\title{
Content Analysis of Psoriasis and Eczema Direct-to-Consumer Advertisements
}

\author{
Alexis Holmes, BA; Cheyenne Williams, BS; Shiyu Wang, MS; Frances K. Barg, PhD, MEd; \\ Junko Takeshita, MD, PhD, MSCE
}

\section{PRACTICE POINTS}

- Racial/ethnic minorities and older adults are underrepresented in direct-to-consumer (DTC) advertisements for psoriasis and eczema.

- Character representation in psoriasis DTC advertisements, in particular, mirrors existing age and racial disparities in treatment with biologics.

- Disease-specific factual content was sparse, and obvious depictions of skin disease and symptoms were uncommon, especially among psoriasis DTC advertisements.

- Dermatologists should be aware of these deficiencies in psoriasis and eczema DTC advertisements and take care not to further reinforce existing knowledge gaps and inequitable treatment patterns among patients.

Direct-to-consumer (DTC) advertisements have been associated with both beneficial and detrimental effects on real-world health care utilization. We aimed to evaluate advertisement placement, character representation, and disease-related content in psoriasis and eczema DTC advertisements. We conducted a content analysis of DTC advertisements televised on 4 major networks over 14 consecutive days in July 2018. In total, 13 advertisements for psoriasis and 27 advertisements for eczema were identified. Among psoriasis advertisements, 81 affected characters were shown, and among eczema advertisements, 80 affected characters were shown. Our findings suggest missed opportunities for education and patient reach among psoriasis and eczema DTC advertisements. Lack of diversity of characters among advertisements for psoriasis and eczema may reinforce existing and emerging treatment disparities.

Cutis. 2020;106:147-150. $\square$ irect-to-consumer (DTC) advertisements are an important and influential source of health-related information for Americans. In 1997, the US Food and Drug Administration (FDA) relaxed regulations and permitted DTC drug advertisements to be televised. Now, via television alone, the average American is exposed to more than 30 hours annually of DTC advertisements for drugs, ${ }^{1}$ which exceeds, by far, the amount of time the average American spends with his/her physician. ${ }^{2}$ The United States spends $\$ 9.6$ billion on DTC advertisements per year, of which $\$ 605$ million is spent exclusively on DTC advertisements for dermatologic conditions-one of the highest amounts of spending for DTC advertisements, second only to diabetes. ${ }^{3}$

The increase in advertising for dermatologic conditions is reflective of the rapid growth in the number of treatment options available for chronic skin diseases, especially psoriasis. Since 2004, 11 biologics and 1 oral medication were FDA approved for the treatment of moderate to severe psoriasis. Despite the expansion of treatment options for psoriasis, knowledge and understanding of psoriasis and its treatments generally are poor, ${ }^{4,5}$ and undertreatment of psoriasis continues to be common. ${ }^{6}$ Data also suggest existing age and racial disparities in psoriasis treatment in the United States, whereby patients who are older or Black are less likely to receive biologic therapies. ${ }^{7-9}$ Although the exact causes of these disparities remain unclear, one study found that Black patients with psoriasis were less familiar with biologics compared to White patients, ${ }^{10}$ which suggests that the racial disparity in biologic treatment of psoriasis could be due to less

From the Perelman School of Medicine, University of Pennsylvania, Philadelphia. Ms. Wang and Dr. Takeshita are from the Department of Dermatology; Drs. Barg and Takeshita are from the Department of Biostatistics, Epidemiology and Informatics; and Dr. Barg also is from the Department of Family Medicine and Community Health.

Ms. Holmes was supported by the Dermatology Foundation Diversity Research Supplement Award. Dr. Takeshita is supported by the National Institute of Arthritis Musculoskeletal and Skin Diseases under Grant K23-AR068433, receives a research grant from Pfizer Inc (to the Trustees of the University of Pennsylvania) for work that is unrelated to this study, and has received payment for continuing medical education work related to psoriasis that was supported indirectly by Eli Lilly and Company and Novartis. Ms. Williams, Ms. Wang, and Dr. Barg report no conflict of interest. Correspondence: Junko Takeshita, MD, PhD, MSCE, University of Pennsylvania Perelman School of Medicine, 3400 Civic Center Blvd, 7th Floor, South Tower, Office 728, Philadelphia, PA 19104 (Junko.Takeshita@pennmedicine.upenn.edu).

doi: 10.12788 /cutis.0070 
exposure to and thus recognition of biologics as treatments of psoriasis among Black patients.

Some data suggest that DTC advertisements may affect drug uptake by encouraging patients to request advertised medications from their medical providers. ${ }^{11,12}$ As such, DTC advertisements are a potentially important source of exposure and information for patients. However, is it possible that DTC advertisements also may contribute to widening knowledge gaps among certain populations, and thus treatment disparities, by neglecting certain groups and targeting others with their content? In an effort to answer this question, we performed an analysis of DTC advertisements for psoriasis and eczema with special attention to advertisement placement, character representation, and diseaserelated content. We specifically targeted advertisements for psoriasis and eczema, as advertisements for the former are rampant and advertisements for the latter are on the rise because of emerging therapies. We hypothesized that age and racial/ethnic diversity among advertisement characters is poor, and disease-related content is lacking.

\section{Materials and Methods}

Study Design and Sample - We performed a cross-sectional analysis of televised DTC advertisements for psoriasis and eczema over 14 consecutive days (July 1, 2018, to July 14, 2018). We accessed Nielsen's top 10 lists, specifically Prime Broadcast Network TV-United States and Prime Broadcast Programs Among African-American, from June 2018 and identified the networks with the greatest potential exposure to American consumers: ABC, CBS, FOX, and NBC. ${ }^{13,14}$ Each day, programming aired from 5 PM to 11 PM EST was recorded on a random selection of 2 of 4 listed networks. No pair of networks was recorded for 2 consecutive days, and each day of the week was represented for each network.

The FDA identifies DTC advertisement types as productclaim, reminder, and help-seeking advertisements. Productclaim advertisements are required to include the following information for the drug of interest: name; at least 1 FDAapproved indication; the most notable risks; and reference to a toll-free telephone number, website, or print advertisement by which a detailed summary of risks and benefits can be accessed. Reminder advertisements include the name of the drug but no information about the drug's use.$^{15}$ Help-seeking advertisements describe a disease or condition without referencing a specific drug treatment. Product-claim, reminder, and help-seeking advertisements for psoriasis or eczema that aired during the recorded time frame were included for analysis; advertisements that aired during sporting events and special programming were excluded.

DTC Advertisement Coding-Advertisement placement (ie, network, day of the week, time, associated television program), type, and target disease were documented for all advertisements included in the study. The content of each unique advertisement for psoriasis and eczema also was documented electronically in REDCap (Research Electronic Data Capture) as follows: characteristics of affected individuals and disease-related content. Advertisement coding was performed independently by 2 graduate students (A.H. and C.W.). First, one-third of the advertisements were randomly selected to be coded by both students. Intercoder agreement between the 2 students was $95.3 \%$. Coding disagreements were primarily due to misunderstanding of definitions and were resolved through consensus. Subsequently, the remaining advertisements were randomly distributed between the 2 students, and each advertisement was coded by 1 student.

Statistical Analysis-All data were summarized descriptively with counts and frequencies using Stata 15 (StataCorp).

\section{Results}

We identified 297 DTC advertisements addressing 25 different conditions during our study period. CBS, $\mathrm{ABC}, \mathrm{NBC}$, and FOX aired $44.4 \%, 26.3 \%, 24.4 \%$, and $5.1 \%$ of advertisements, respectively. Overall, DTC advertisements were least likely to air on Saturdays and between the hours of 5 PM and 6 PM on any day. Product-claim advertisements accounted for $83.2 \%$ of DTC advertisements, $15.8 \%$ were help-seeking advertisements, and the remaining 1.0\% were reminder advertisements. Advertisements for skin conditions represented $16.5 \%(n=49)$ of all DTC advertisements, of which $81.6 \%(n=40)$ were for psoriasis or eczema, while the other $18.4 \%(n=9)$ were for hyperhidrosis. In total, 13 advertisements for psoriasis and 27 advertisements for eczema were aired during the study period.

Psoriasis DTC Advertisements-There were 5 unique psoriasis DTC advertisements, all of which were product-claim advertisements, with 1 each for secukinumab (Cosentyx [Novartis]), ixekizumab (Taltz [Eli Lilly and Company]), and guselkumab (Tremfya [Janssen Biotech, Inc]), and 2 for adalimumab (Humira [AbbVie Inc]). The advertisements aired on $\mathrm{ABC}(\mathrm{n}=5[38.5 \%]), \mathrm{CBS}(\mathrm{n}=5[38.5 \%])$, and NBC $(n=3$ [23.1\%]). Most advertisements aired on weekdays $(61.5 \%)$ between 6 PM and 7 PM (69.2\%) and during news programming $(69.2 \%)$.

Psoriasis Character Portrayal and Disease-Related ContentWe identified 81 main characters who were depicted as having psoriasis among all advertisements. Characteristics of the affected characters are summarized in the Table. All affected characters were perceived to be younger adults, and there was a slight female predominance (58.0\% [47/81]). Most characters were perceived to be White (92.6\% [75/81]). Black and Asian characters only represented 6.2\% (5/81) and $1.2 \%$ (1/81) of all affected individuals, respectively. Notably, the advertisements that featured only White main characters were aired 2.75 times more frequently than the advertisements that included non-White characters.

Psoriasis was shown on the skin of at least 1 character in an obvious depiction (ie, did not require more than 1 viewing) in $84.6 \%(11 / 13)$ of the advertisements. Symptoms of psoriasis (communicated either verbally or visually) were included in only $15.4 \%(2 / 13)$ of advertisements. No advertisements included information on the epidemiology of (ie, prevalence, subpopulations at risk), risk factors for, pathophysiology of, or comorbid diseases associated with psoriasis. 


\section{Main Character Depiction in Direct-to- Consumer Advertisements by Exposure}

\begin{tabular}{lll}
\hline & $\begin{array}{l}\text { Psoriasis, } \mathrm{n}(\%) \\
(\mathrm{N}=81)\end{array}$ & $\begin{array}{l}\text { Eczema, } \mathrm{n}(\%) \\
(\mathrm{N}=80)\end{array}$ \\
\hline $\begin{array}{l}\text { Age group } \\
\text { Infant }\end{array}$ & $0(0)$ & $0(0)$ \\
\hline Child & $0(0)$ & $53(66.3)$ \\
\hline Teenager & $0(0)$ & $0(0)$ \\
\hline Younger adult & $81(100)$ & $27(33.8)$ \\
\hline Older adult & $0(0)$ & $0(0)$ \\
Gender & $34(42.0)$ & $23(28.8)$ \\
\hline Male & $47(58.0)$ & $57(71.3)$ \\
\hline Female & & \\
Race/ethnicity & $75(92.6)$ & $43(53.8)$ \\
\hline White & $5(6.2)$ & $23(28.8)$ \\
\hline Black & $1(1.2)$ & $14(17.5)$ \\
\hline Asian & $0(0)$ & $0(0)$ \\
\hline Hispanic & $0(0)$ & $0(0)$ \\
\hline Other & $0(0)$ & $0(0)$ \\
\hline Unknown & $22(27.2)$ & $43(53.8)$ \\
\hline Skin condition shown & $(13.6)$ & \\
\hline Not shown & $48.8)$ \\
\hline
\end{tabular}

Eczema DTC Advertisements-Among the 27 eczema advertisements aired, there were 4 unique advertisements, of which 3 were product-claim advertisements (all for crisaborole [Eucrisa (Pfizer Inc)]), and 1 was a help-seeking advertisement that was sponsored by Sanofi Genzyme and Regeneron Pharmaceuticals. The advertisements aired on $\operatorname{ABC}(n=2[7.4 \%]), \operatorname{CBS}(n=17[63.0 \%])$, and NBC $(n=8$ [29.6\%]). All advertisements aired on weekdays between 7 $\mathrm{PM}$ and $8 \mathrm{PM}$ and during non-news programming.

Eczema Character Portrayal and Disease-Related ContentWe identified 80 main characters who were depicted to be affected by eczema among all advertisements. Characteristics of the affected characters are summarized in the Table. Most of the affected characters were perceived to be White (53.8\% [43/80]) and female (71.3\% [57/80]). Other races depicted included Black (28.8\% [23/80]) and Asian (17.5\% [14/80]). Each unique eczema advertisement included at least 1 non-White main character. Most eczema main characters were perceived to be children (66.3\% [53/80]), followed by younger adults (33.8\% [27/80]). No infants, teenagers, or older adults were shown as being affected by eczema.

Skin manifestations of eczema were portrayed on at least 1 character in all of the advertisements; $77.8 \%$ (21/27) of the advertisements had at least 1 obvious depiction. Symptoms of eczema and the mechanism of disease (pathophysiology) were each included in $44.4 \%$ (12/27) of advertisements. This information was included exclusively in the single helpseeking advertisement, which also referenced a website for additional disease-related information. No advertisements included information on the epidemiology of, risk factors for, or comorbid diseases associated with eczema.

\section{Comment}

In our study of televised DTC advertisements for psoriasis and eczema in the United States, we identified underrepresentation of racial/ethnic minorities and specific age groups (older adults for psoriasis and all adults for eczema) across all advertisements. Although psoriasis is suggested to be less prevalent among minority patients $(1.3 \%-1.9 \%$ among Black patients and 1.6\% among Hispanic patients) compared to White patients $(2 \%-4 \%),{ }^{16,17}$ minority vs White representation in psoriasis DTC advertisements was disproportionately lower than population-based prevalence estimates. Direct-to-consumer advertisements for eczema included more minority characters than psoriasis advertisements; however, minority representation remained inadequate considering that childhood eczema is more prevalent among Black vs White children, ${ }^{18}$ and adult eczema is at least as prevalent among minority patients compared to White patients. ${ }^{19}$ Not only was minority representation in all advertisements poor, but advertisement placement also was suboptimal, particularly for reaching Black viewers. FOX network was home to 2 of the top 3 primetime broadcast programs among Black viewers around the study period, ${ }^{13}$ yet no DTC advertisements were aired on FOX. Together, our findings suggest inadequate patient reach of psoriasis and eczema DTC advertisements that, particularly in the case of psoriasis, mirror and may reinforce existing age and racial disparities in treatment ${ }^{5-7}$ and poor familiarity with biologics. ${ }^{10} \mathrm{~A}$ similar impact of eczema DTC advertisements on emerging eczema treatment patterns and potential treatment disparities also might be anticipated and is of concern.

The current literature regarding minority representation in DTC advertisements is mixed. Some studies report underrepresentation of Black and other minority patients across a variety of diseases. ${ }^{20}$ Other studies suggest that representation of Black patients, in particular, generally is adequate, except among select serious health conditions, and that advertisements depict tokenism or stereotypical roles for minorities. ${ }^{21}$ Our study provides new and specific insight about the state of racial/ethnic and age diversity, or lack thereof, in DTC advertisements for the skin conditions that currently are most commonly targeted-psoriasis and eczema. Although it remains unclear whether DTC advertisements are good or bad, existing data suggest that potential benefits of DTC advertisements include strengthening of patient-provider relationships, reduction of underdiagnosis and undertreatment of disease, and reduction of disease stigma. ${ }^{22}$ However, in our analyses, we found diseasespecific factual content among all DTC advertisements to 
be sparse and obvious depictions of skin disease and symptoms to be uncommon, especially for psoriasis. As such, it seems unlikely that existing DTC advertisements for psoriasis and eczema can be expected to contribute to meaningful disease education, reduce underdiagnosis, and reduce the stigmatizing attitudes that have been documented for both skin diseases. ${ }^{23-25}$

Furthermore, it is important to consider our findings in light of the role that social identity theory plays in marketing. Social identity theory supports the idea that a person's social identity (eg, age, gender, race/ethnicity) influences his/her behavior, perceptions, and performance. ${ }^{26}$ The principle of homophily - the tendency for individuals to have positive ties to those who are similar to themselves-is a critical concept in social identity theory and suggests that consumers are more likely to pay attention to and be influenced by sources perceived as similar to themselves.$^{20}$ Thus, even if the potential benefits of DTC advertisements were to be realized for psoriasis and eczema, the lack of adequate minority and older adult representation raises concerns about whether these benefits would reach a diverse population and if the advertisements might further potentiate existing knowledge and treatment disparities.

Limitations-Our study is not without limitations. The sampling period was short and might not reflect advertisement content over a longer time course. We did not evaluate other potential sources of information, such as the Internet and social media. Nevertheless, televised DTC advertisements remain a major source of medical and drug information for the general public. We did not directly evaluate viewers' reactions to the DTC advertisements of interest; however, other literature lends support to the significance of social identity theory and its impact on consumer behavior. ${ }^{26}$

\section{Conclusion}

Our study highlights a lost opportunity among psoriasis and eczema DTC advertisements for patient reach and disease education that may encourage existing and emerging knowledge and treatment disparities for both conditions. Our findings should serve as a call to action to pharmaceutical companies and other organizations involved in creating and supporting DTC advertisements for psoriasis and eczema to increase the educational content, diversify the depicted characters, and optimize advertisement placement.

\section{REFERENCES}

1. Brownfield ED, Bernhardt JM, Phan JL, et al. Direct-to-consumer drug advertisements on network television: an exploration of quantity, frequency, and placement. J Health Commun. 2004;9:491-497.

2. Tai-Seale M, McGuire TG, Zhang W. Time allocation in primary care office visits. Health Serv Res. 2007;42:1871-1894.

3. Schwartz LM, Woloshin S. Medical marketing in the United States, 19972016. JAMA. 2019;321:80-96.

4. Lanigan SW, Farber EM. Patients' knowledge of psoriasis: pilot study. Cutis. 1990;46:359-362.

5. Renzi C, Di Pietro C, Tabolli S. Participation, satisfaction and knowledge level of patients with cutaneous psoriasis or psoriatic arthritis. Clin Exp Dermatol. 2011;36:885-888.
6. Lebwohl MG, Bachelez H, Barker J, et al. Patient perspectives in the management of psoriasis: results from the population-based Multinational Assessment of Psoriasis and Psoriatic Arthritis Survey. J Am Acad Dermatol. 2014;70:871-881.e871-830.

7. Wu JJ, Lu M, Veverka KA, et al. The journey for US psoriasis patients prescribed a topical: a retrospective database evaluation of patient progression to oral and/or biologic treatment. J Dermatolog Treat. 2019;30:446-453.

8. Takeshita J, Gelfand JM, Li P, et al. Psoriasis in the US Medicare population: prevalence, treatment, and factors associated with biologic use. J Invest Dermatol. 2015;135:2955-2963.

9. Kerr GS, Qaiyumi S, Richards J, et al. Psoriasis and psoriatic arthritis in African-American patients - the need to measure disease burden. Clin Rheumatol. 2015;34:1753-1759.

10. Takeshita J, Eriksen WT, Raziano VT, et al. Racial differences in perceptions of psoriasis therapies: implications for racial disparities in psoriasis treatment. J Invest Dermatol. 2019;139:1672-1679.e1.

11. Wu MH, Bartz D, Avorn J, et al. Trends in direct-to-consumer advertising of prescription contraceptives. Contraception. 2016; 93:398-405.

12. Mintzes B, Barer ML, Kravitz RL, et al. How does direct-to-consumer advertising (DTCA) affect prescribing? a survey in primary care environments with and without legal DTCA. CMAJ. 2003;169:405-412.

13. Topten. Nielson website. https://www.nielsen.com/us/en/top -ten/. Accessed July 22, 2020.

14. Leading ad supported broadcast and cable networks in the United States in 2019, by average number of viewers. Statistia website. https://www.statista.com/statistics/530119/tv-networks-viewers-usa/. Accessed July 22, 2020.

15. Prescription drug advertisements. Electronic Code of Federal Regulations website. https://www.ecfr.gov/cgi-bin/text-idx?SID=d4f 308e364578bda8e55a831638a26c6\&mc $=$ true \&node $=$ pt21.4.202\&rgn $=$ div5. Updated August 12, 2020. Accessed August 12, 2020.

16. Gelfand JM, Stern RS, Nijsten T, et al. The prevalence of psoriasis in African Americans: results from a population-based study. J Am Acad Dermatol. 2005;52:23-26.

17. Rachakonda TD, Schupp CW, Armstrong AW. Psoriasis prevalence among adults in the United States. J Am Acad Dermatol. 2014; 70:512-516.

18. Centers for Disease Control and Prevention. National Center for Health Statistics, National Health Interview Survey, 2014. https://www.cdc.gov /nchs/data/health_policy/eczema_skin_problems_tables.pdf. Accessed July 22, 2020

19. Chiesa Fuxench ZC, Block JK, Boguniewicz M, et al. Atopic dermatitis in America study: a cross-sectional study examining the prevalence and disease burden of atopic dermatitis in the US adult population. J Invest Dermatol. 2019;139:583-590.

20. Welch Cline RJ, Young HN. Marketing drugs, marketing health care relationships: a content analysis of visual cues in direct-toconsumer prescription drug advertising. Health Commun. 2004; 16:131-157.

21. Ball JG, Liang A, Lee WN. Representation of African Americans in direct-to-consumer pharmaceutical commercials: a content analysis with implications for health disparities. Health Mark $Q$. 2009;26:372-390.

22. Ventola CL. Direct-to-consumer pharmaceutical advertising: therapeutic or toxic? P T. 2011;36:669-674, 681-684.

23. Pearl RL, Wan MT, Takeshita J, et al. Stigmatizing attitudes toward persons with psoriasis among laypersons and medical students. J Am Acad Dermatol. 2019;80:1556-1563.

24. Chernyshov PV. Stigmatization and self-perception in children with atopic dermatitis. Clin Cosmet Investig Dermatol. 2016;9:159-166.

25. Wittkowski A, Richards HL, Griffiths CEM, et al. The impact of psychological and clinical factors on quality of life in individuals with atopic dermatitis. J Psychosom Res. 2004;57:195-200.

26. Forehand MR, Deshpande R, Reed 2nd A. Identity salience and the influence of differential activation of the social self-schema on advertising response. J Appl Psychol. 2002;87:1086-1099. 